\title{
The Influence of the Great Hanshin Earthquake on Human Response to Environmental Vibration Due to the Shinkansen
}

\author{
Satokazu SUMITOMO ${ }^{1 *}$, Saburomaru TSUJIMOTO', \\ Setsuo MAEDA ${ }^{2}$ and Yasutoshi KITAMURA ${ }^{3}$
}

\footnotetext{
${ }^{1}$ The Hyogo Prefectural Institute of Environmental Science, 3-1-27 Yukihira-cho Suma-ku, Kobe 654-0037, Japan

${ }^{2}$ Department of Industrial Engineering, Kinki University, 3-4-1 Kowakae, Higashi-Osaka 577-8502, Japan

${ }^{3}$ Department of Civil Engineering, Kobe University, Rokkodai, Nada-ku, Kobe 657-0013, Japan
}

Received April 6, 1998 and accepted May 20, 1998

\begin{abstract}
A severe earthquake of magnitude 7.2 hit the west part of Japan on January 17, 1995. A part of the Shinkansen railway, which is one of the most popular high-speed mass transportation systems in Japan, was seriously damaged by the earthquake. About 80 days later, the Shinkansen service was resumed but complaints about vibration due to the passing Shinkansen increased rapidly among residents near the tracks. This paper reports the results of two investigations that were carried out in both stricken and non-stricken areas to determine the cause of complaint. In the first investigation, the ground vibration propagation induced by passing trains was measured. In the second investigation, questionnaires were distributed to the people living near the Shinkansen tracks. As a result, it was found out that the vibration levels before and after the earthquake were almost the same at most measured points in the stricken area. It was also found that the vibration levels in the stricken area and a non-stricken area were almost the same within $50 \mathrm{~m}$ from the Shinkansen tracks. However the results of the questionnaire survey showed that people's nuisance due to the vibration in the stricken area was clearly greater than that in the non-stricken area. This inconsistency was explained using the "category judgment method", which is generally used to determine the relationship between a physical stimulus and psychological reaction. According to the results of this analysis, the vibration level, at which $50 \%$ of the inhabitants complained about Shinkansen vibration, was approximately $54 \mathrm{~dB}$ in the non-stricken area and $50 \mathrm{~dB}$ in the stricken area. This result suggests that the people who experienced the severe earthquake became $4 \mathrm{~dB}$ more sensitive to the Shinkansen vibration than the people living in a non-stricken area despite the fact that this investigation was carried out 10 months after the earthquake struck.
\end{abstract}

Key words: Vibration, Human response, Shinkansen, Earthquake, Category judgement

\section{Introduction}

On January 17, 1995, a great earthquake hit the west part of Japan (the southern part of Hyogo Prefecture). The ground vibration observed in neighboring districts exceeded 500

*To whom correspondence should be addressed. gals in maximum vertical acceleration ${ }^{1,2)}$. There were more than 6,000 casualties, and the Shinkansen railway also suffered damage. The most severely affected area was along the 50-km stretch from Shin-Osaka to Akashi, where railway bridges collapsed and piers were severely damaged in more than 10 places, as shown in Fig. 1.

As the Shinkansen railway plays an important role in 


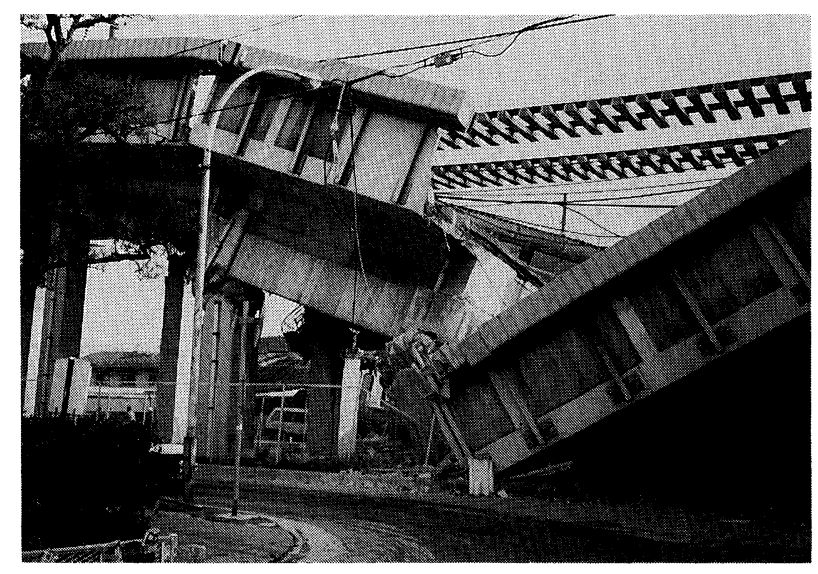

Fig. 1. A collapsed section of Shinkansen railway

supporting the Japanese economy, priority was given to restoration work, and services were resumed about 80 days later. However, after the resumption of services, the number of complaints about vibration due to Shinkansen trains increased rapidly among the inhabitants of the stricken area.

This research was carried out to investigate the cause of these complaints. Two hypothesis were considered. One was that the earthquake caused changes in the ground or indoor vibration conditions, and the other was that experience of the earthquake caused people to become more sensitive to vibration.

In this work, two types of study were carried out in the stricken area and a non-stricken area. A ground vibration investigation was performed to obtain information on the changes in vibration propagation in the stricken area and non-stricken area, and a questionnaire was used to obtain the opinions of people living close to the tracks regarding the vibration after the Shinkansen resumed service.

The study showed that the sensitivity to vibration of the people who experienced the great earthquake had changed.

\section{Investigation Sites}

Studies were carried out in the stricken area and a nonstricken area. A part of the stricken area (a site in Nishinomiya city, hereafter referred to as Site A) was selected as the study area. Most of the dwellings in this area suffered damage, and $30 \%$ of them collapsed completely. A part of Aioi city (hereafter referred to as Site B) (approximately $100 \mathrm{~km}$ away from Site A) in which there was hardly any damage to dwellings or to the Shinkansen railway, was selected as the reference area. The locations of Site A and Site B are shown

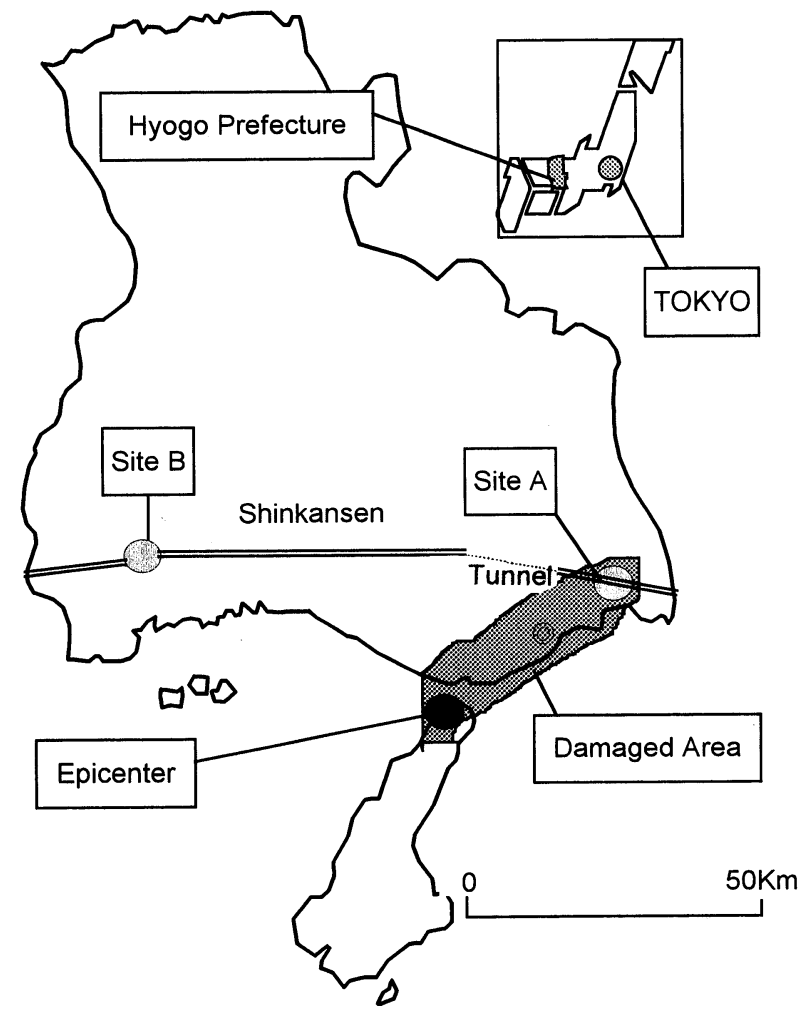

Fig. 2. Measurement sites and damaged area

in Fig. 2. Both sites are almost entirely residential areas and most of the dwellings are two-storied wooden houses. The Shinkansen railway in these areas is an elevated structure made of reinforced concrete. The height and width of the structure are 8-10 m and $10 \mathrm{~m}$, respectively. In both sites, the houses closest to the Shinkansen railway are $15 \mathrm{~m}$ from the railway tracks.

\section{Ground Vibration Measurement}

In order to obtain information on the changes in ground vibration propagation, two measurements were performed. The first involved a comparison of the vibration levels before and after the earthquake in the stricken area. The other involved a comparison of the ground vibration level after the earthquake between Site A and Site B.

The first measurement was performed to determine vertical vibration levels at 29 sites in the stricken area which have been measured continuously, since before the earthquake. The measurement points at each site are $12.5 \mathrm{~m}$ and $25 \mathrm{~m}$ from the Shinkansen tracks.

The second measurement was performed to determine characteristics of vibration attenuation with distance at Site 


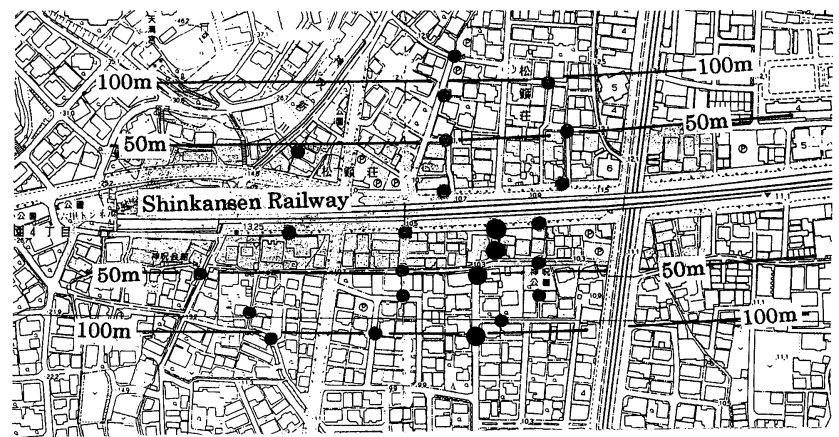

Site A

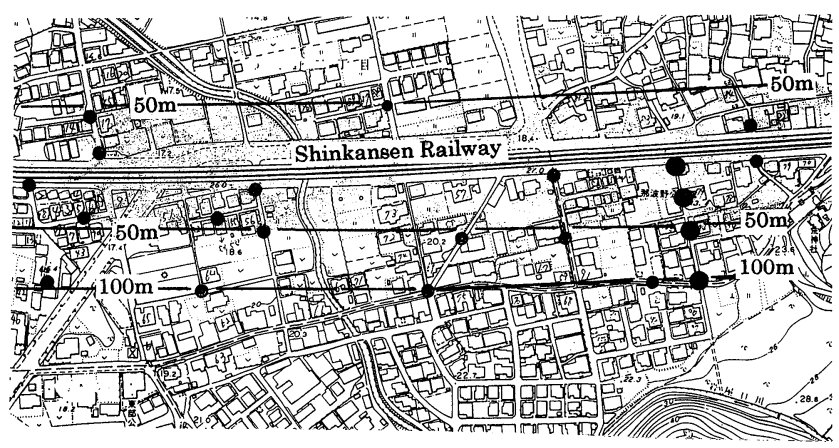

Site B

Fig. 3. Measurement points at Site A and Site B

: Measurement points for vibration attenuation with distance

- : Measurement points for distribution

A and Site B. The measurement points were located 12.5 $\mathrm{m}, 25 \mathrm{~m}, 50 \mathrm{~m}$ and $100 \mathrm{~m}$ from the tracks. In addition to obtain information on the distribution of ground vibration level at Site A and Site B, measurements were made at 20 points at each site. In order to determine the relationship between outdoor and indoor vibration, measurements were made at 5 houses at each site.

\section{Method of measurement}

Fig. 3 shows the locations of measurement points at Site A and Site B. Black circles on the map are measurement points for ground vibration.

All ground vibration measurement points were on roads perpendicular to the Shinkansen railway. At each point, pickup sensors were placed on the road surface. Indoor vibration was measured in the center of a room on the first floor of each house, and outdoor vibration was measured on the ground in front of the house.

The measurement instruments and system are shown in Fig. 4. The vibration levels were measured by a three-axis accelerometer (RION, PV83-A). The output signals from this accelerometer were input to a vibration-level meter and

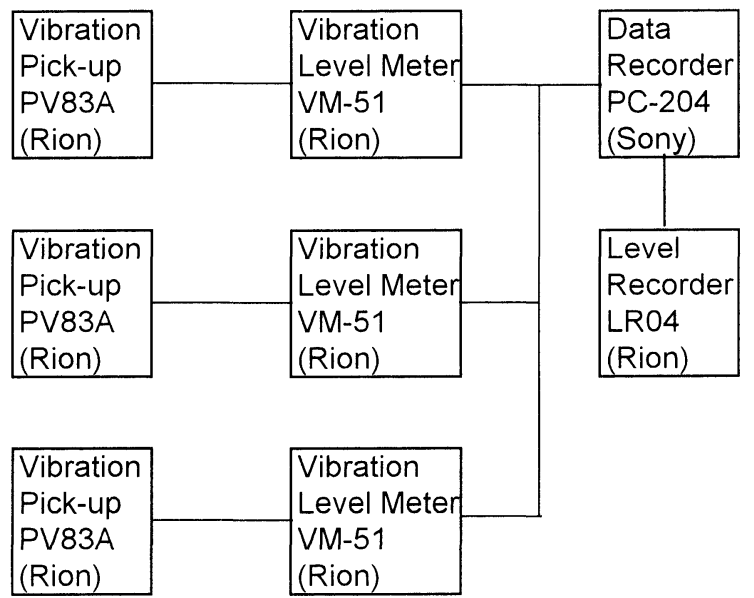

Fig. 4. Vibration measurement instruments and system

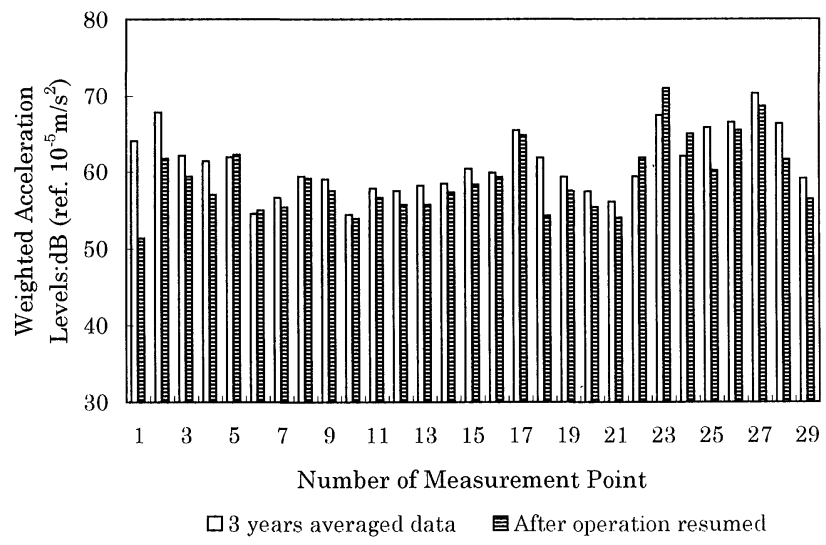

Fig. 5. Comparison of the vertical weighted acceleration levels in the stricken area between last 3 years averaged data and data after operation resumed at $12.5-\mathrm{m}$ Point

data recorder. Vertical acceleration levels were measured on the ground surface and on the first floor in the houses. The vibration-level meter conforms to JIS C $1510^{3)}$ and its weighted filter conforms to ISO $8041^{4)}$.

The vibrations caused by the Shinkansen were measured for 20 trains passing by the measurement points. Indoor vibration and distribution were measured for 10 trains at each point of each area. The vibration levels for each measurement were determined using the method stipulated by Japan Environmental Agency (average of the upper half trains) ${ }^{5}$.

\section{Results of the ground vibration measurement}

Fig. 5 shows the results of the comparison between the weighted vertical acceleration level (hereafter vibration level) averaged over the last three year's data and the data measured 


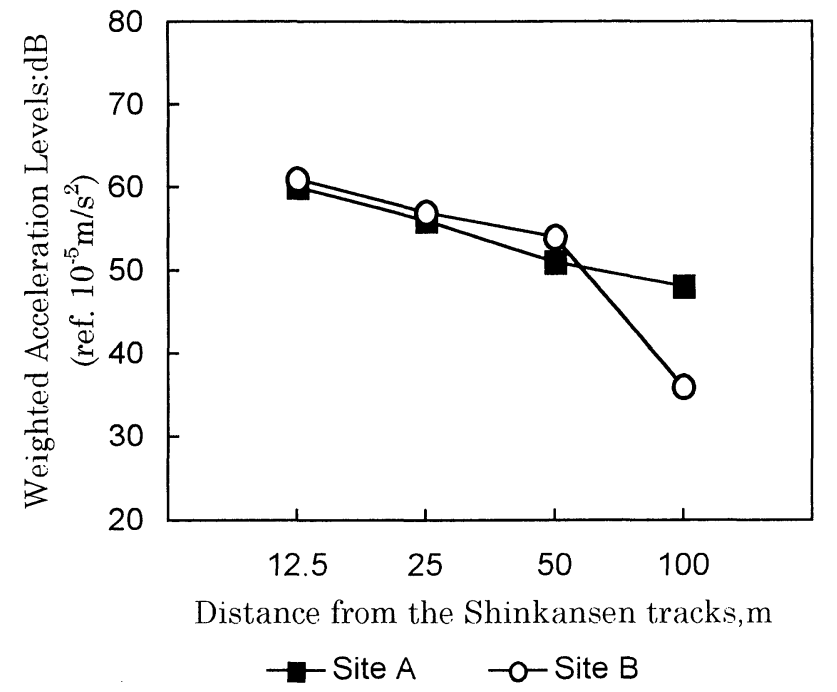

Fig. 6. Attenuation of vertical weighted acceleration levels with distance

after operation resumed. According to the result, for the point $12.5 \mathrm{~m}$ from the Shinkansen tracks, only 3 sites had higher vibration level than before the earthquake, and 26 sites had almost the same or smaller vibration level than before. Point number 1 showed a decrease of more than $10 \mathrm{~dB}$, but there was no clear reason for this result. Similar results were obtained at the 25-m point.

Fig. 6 shows the ground vibration attenuation with distance at Site $\mathrm{A}$ and Site B. The vibration levels at both sites were almost the same for distances of $12.5 \mathrm{~m}$ and $50 \mathrm{~m}$ from the Shinkansen tracks. For the $100-\mathrm{m}$ point, the vibration level at Site A was greater than that at Site B.

Table 1 shows a comparison of the indoor (on the $1 \mathrm{st}$ floor) and outdoor (ground surface) vibration levels measured for five houses at each site. The average level of indoor and outdoor vibration at both sites was almost the same.

\section{Questionnaire Survey}

\section{Distribution of questionnaire}

A questionnaire survey was conducted at Site A and Site $B$ to examine the degree of annoyance concerning vibration due to the Shinkansen. In each area shown in Fig. 3, 100 samples were taken; 50 samples within the $0-50 \mathrm{~m}$ area and 50 samples within the $50-100 \mathrm{~m}$ area from the Shinkansen tracks. The questionnaire survey was conducted through interviews in which basic data on the interviewees and their opinions about vibration due to the Shinkansen were obtained. Thirty percent of the interviewees were over
Table 1. Indoor and outdoor weighted acceleration levels

\begin{tabular}{ccccc}
\hline No. & Outdoor & Indoor & Dif. & Distance \\
\hline A1 & 64 & 61 & -3 & 15 \\
A2 & 60 & 65 & 5 & 15 \\
A3 & 60 & 60 & 0 & 30 \\
A4 & 57 & 59 & 2 & 30 \\
A5 & 50 & 57 & 7 & 40 \\
B1 & 58 & 67 & 9 & 15 \\
B2 & 57 & 58 & 1 & 15 \\
B3 & 54 & 59 & 5 & 20 \\
B4 & 47 & 46 & -1 & 50 \\
B5 & 52 & 55 & -3 & 50 \\
\hline
\end{tabular}

$\mathrm{dB}\left(\right.$ ref. $10^{-5} \mathrm{~m} / \mathrm{s}^{2}$ )

A1-A5: Site A, B1-B5: Site B.

Dif.: difference between indoor and outdoor vibration levels. Distance: distance from the Shinkansen tracks, $m$.

60 years of age, and interviewees in their 30's, 40's and 50 's each accounted for $20-25 \%$. Approximately $80 \%$ of the interviewees were women. There was no noteworthy difference in answers between men and women.

\section{Questionnaire}

Each interviewee was asked to respond to each of the questions by selecting one of five levels of annoyance as follows:-

1. very annoyed 2 . annoyed 3 . fairly annoyed 4. slightly annoyed 5. not annoyed at all.

The following questions regarding the effect of vibration due to the Shinkansen were asked.

a. How do you describe your annoyance due to the Shinkansen vibration?

b. How do you feel about the vibration of doors and windows?

c. How do you feel about the vibration of tableware and decorations?

d. How do you feel about the vibration of furniture?

e. How do you describe your irritation?

f. How do you describe the interruption to reading, writing or concentrating?

g. How do you describe the interruption of your sleep?

h. How do you describe the annoyance of watching television and listening to radio?

i. Have you become more sensitive to the Shinkansen vibration since the earthquake? (To this question, the answer is either YES or NO)

\section{Results of the questionnaire survey}

Results of the questionnaire were classified by a simple 


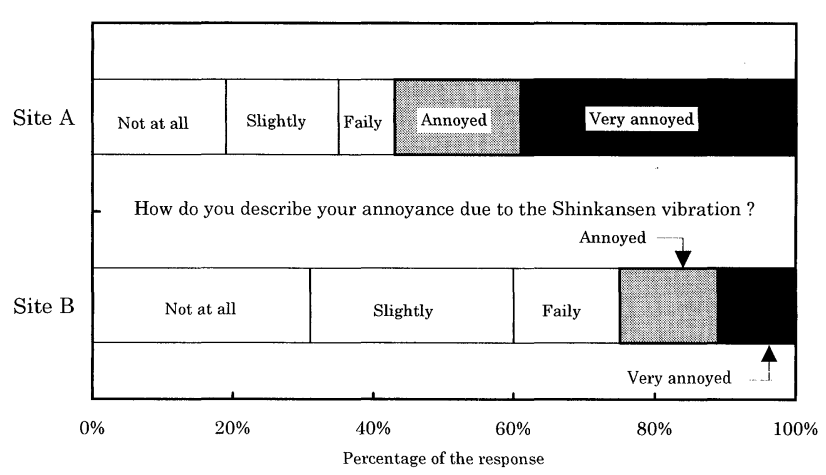

Fig. 7. Degree of annoyance due to the Shinkansen vibration

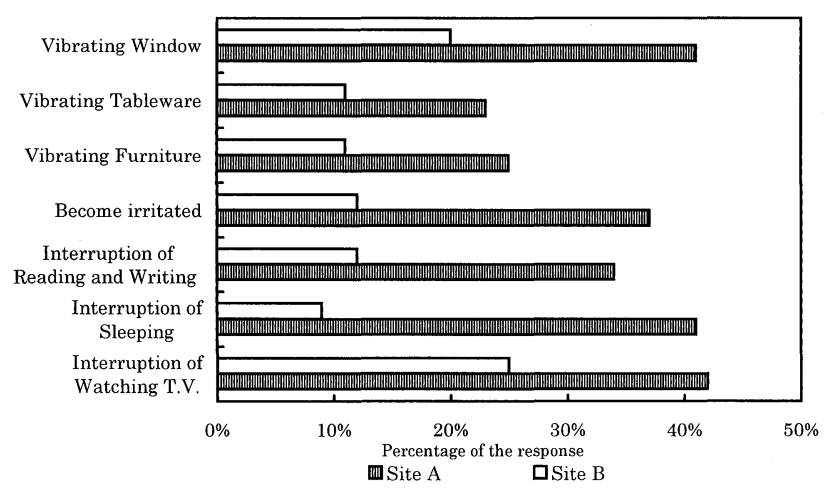

Fig. 8. Sum of responses of very annoyed and annoyed due to the Shinkansen vibration

gathering method. Fig. 7 shows the degrees of annoyance due to the Shinkansen vibration. The percentage of the sum of "very annoyed" and "annoyed" responses was 57\% for Site A and $25 \%$ for Site B. The pecentage of the sum of "very annoyed" and "annoyed" responses to each question is shown in Fig. 8. As an example, the case of "Annoyed by window vibration", the percentage of responses is $41 \%$ for Site A and $20 \%$ for Site B. It can be concluded generally that the degree of annoyance at Site A was greater than that at Site B, especially in terms of psychological influences such as interruptions of reading, sleep and so on. The results of a question concerning sensitivity to the Shinkansen vibration are shown in Fig. 9. About $70 \%$ of the people at Site A thought that they became more sensitive to the Shinkansen vibration after the earthquake, but at site B, the corresponding fraction was only $16 \%$.

\section{Discussion}

Several results were obtained from ground vibration

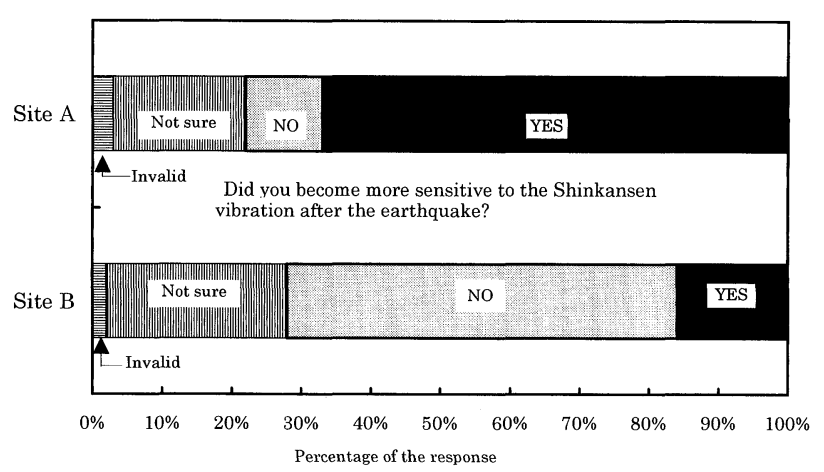

Fig. 9. A comparison of sensitivity of people living at Site A and Site $B$ to the Shinkansen vibration

measurements and the questionnaire.

First, the vertical vibration level after the earthquake in the stricken area was almost the same or smaller than that before the earthquake, as it is shown in Fig. 5. Moreover, the vertical vibration attenuation tendency within $50 \mathrm{~m}$ from the tracks was almost the same at Site A and site B. Moreover, the level of complaints in the stricken area was contrary to this result; that is, after the earthquake, people felt that the vibration level was higher than before.

Second, according to the questionnaire results shown in Figs. 7-9, the degree of annoyance for all items due to the Shinkansen vibration at Site A was greater than that at Site B. The nuisance such as irritation, interruption to reading and writing, and sleep disturbance were particularly marked. However, the results of ground vibration measurement and comparison of indoor and outdoor vibration were almost the same for Site A and Site B.

In order to clarify the cause of this inconsistency, the "category judgment method ${ }^{(6) ", ~ w h i c h ~ i s ~ o n e ~ o f ~ t h e ~ " m e t h o d s ~}$ of successive categories", was adopted to examine the relationship between the questionnaire answers and physical quantities (vibration levels).

\section{Category judgement method}

The category judgment method is employed to determine the relation between a physical stimulus and psychological reaction $^{7,8)}$. In this method, each stimulus is assigned to one of a limited number of categories that differ quantitatively along a defined continuum (for example, annoyance or not annoyance). No assumption is made concerning the psychological equality of category intervals. It is simply assumed that the categories are in correct rank order and that their boundary lines are stable except for sampling errors. It is also assumed that the distribution of psychological 
reaction to one stimulus is a normal distribution on a psychological continuum. The scaling problem is to estimate the values of the categories, or of their limits, along the psychological continuum, and to derive interval-scale measurements of stimuli from these reference values. The calculation procedure is as follows:

$R i(i=1-n)$ is a physical stimulus to be judged, where $i$ is the rank of the stimulus, and $j(j=l-g)$ is the judgment category. $M i$ is the number of the residents who receive stimulus $R i$. Mi people judge such physical stimuli to each category respectively. As a result, we can obtain $A i j$, the frequency distribution of judgment to category $j$ for stimulus Ri.

$$
B_{i j}=\left(\sum_{k=1}^{j} A_{i k}\right) / M_{i}
$$

Using equation (1), frequency distribution $A i j$ is converted to accumulation ratio $B i j$ to category $j$ for $R i$. Also, to obtain the crow flies from psychological average for each stimulus to the upper limit of each category, deviation ratio $Z i j$ ( $j=2$ $g-1)$ corresponding to accumulation ratio $B i j$ is obtained using equation (2), which describes a Gaussian curve.

$$
B_{i j}=\int_{-\infty}^{Z_{i j}} \frac{1}{\sigma \sqrt{2 \pi}} e^{-x^{2} / 2 \sigma^{2}} d x
$$

Except for data for which the deviation ratio cannot be obtained an estimated value, which is the width of adjacent categories is obtained using equation (3).

$$
d_{i j}=Z_{i j}-Z_{i(j-1)}(j=2-g-1)
$$

$M D j$, the average estimated value of a category width is obtained for every category using equation (4). Then using $M D j$, an upper limit scale value $L C j$ is obtained using equation (5),

$$
\begin{aligned}
& M D_{j}=\left(\sum_{k=1}^{i} d_{k j}\right) / i \\
& L C_{j}=\sum_{k=2}^{j} M D_{k}(j=2-g-1)
\end{aligned}
$$

$M C j$ is a middle of every interval of the category, is obtained using equation (6) as a typical value for each category.

$$
M C_{j}=\left(L C_{(j-1)}+L C_{j}\right) / 2
$$

Category $g_{1 / 2}$ which is center of the category is considered as 0 , using equation (7), $M C j$ is converted into a psychological scale value $A C j$.

$$
A C_{j}=M C_{j}-M C_{8_{1 / 2}}
$$

Scale value $M D N i$, which is the median of the frequency distribution for each stimulus, is obtained using $L C j$ and $B i j$.

$$
M D N_{i}=L C_{(j-1)}+\frac{L C_{j}-L C_{(j-1)}}{B_{i j}-B_{i(j-1)}} \times\left(0.5-B_{i(j-1)}\right)
$$

$M C g_{1 / 2}$ is subtracted from $M D N i$, which is converted into a value $M A i$ on the $\mathrm{AC}$ scale.

$$
M A_{i}=M D N_{i}-M C_{g_{1 / 2}}
$$

This $M A i$ on the AC scale shows a value that the people of $50 \%$ of $M i$ person for each stimulus $R i$ evaluated psychologically.

\section{Application of "category judgment method" to the questionnaire}

The category judgment method, based on the results of the questionnaire and the estimated vibration level assigned to residents, was adopted concerning the estimated value of the questionnaire "the degree of the annoyance due to Shinkansen vibration". The stimuli were divided at $5 \mathrm{~dB}$ intervals into 7 phases from $40 \mathrm{~dB}$ to $65 \mathrm{~dB}$.

Fig. 10 shows the relation between the psychological score $(M A i)$ and the vibration level for "the degree of the annoyance due to Shinkansen vibration" at Site A, and Site B. In each figure, $A-G$ is a rank of vibration level as a stimulus; that is A: $65-70 \mathrm{~dB}, \mathrm{~B}: 60-65 \mathrm{~dB}, \mathrm{C}: 55-60 \mathrm{~dB}, \mathrm{D}: 50-55 \mathrm{~dB}, \mathrm{E}$ : 45-50 dB, F: 40-45 dB, G: 35-40 dB. Every position on the vertical axis of $A-G$ shows that $50 \%$ of the people who received the same stimulus (vibration level) evaluated it as the same psychological rank. The regression line in the figure was obtained by the least-squares method for the relationship between psychological position and vibration level rank of A-G which is classified in same interval on the horizontal axis. The regression line gradients for Site A and Site B are almost the same. The intersection point between the regression line and psychological center is taken as the vibration level. From Fig. 10, it can be seen that the vibration level for Site A is approximately $50 \mathrm{~dB}$ and that for Site B is $54 \mathrm{~dB}$.

The above result shows that $50 \%$ of the people complaint against the Shinkansen vibration is about $50 \mathrm{~dB}$ in Site A but about $54 \mathrm{~dB}$ in Site B, that is, people at Site A are approximately $4 \mathrm{~dB}$ more sensitive than people at Site B. This result shows that people living in the stricken area became more sensitive due to the severe earthquake.

\section{Conclusion}

The findings of this investigation are summarized as follows: 


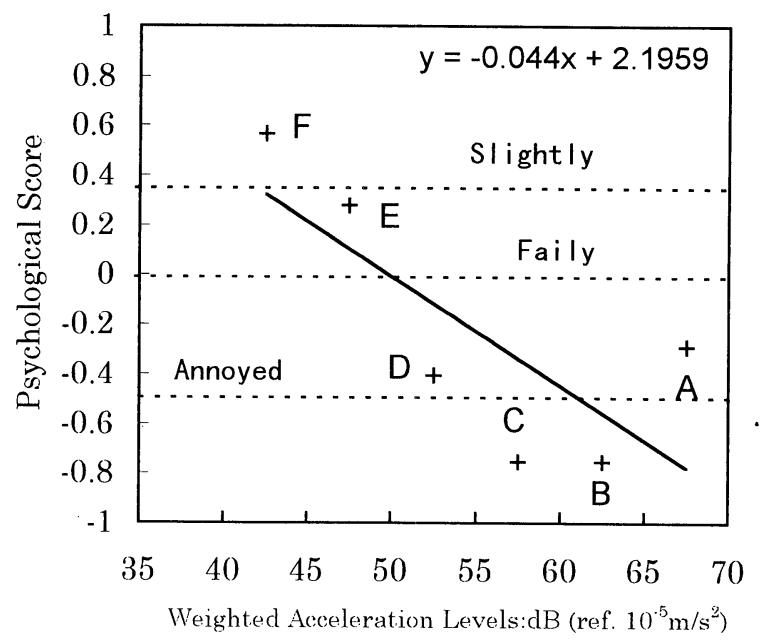

Site A

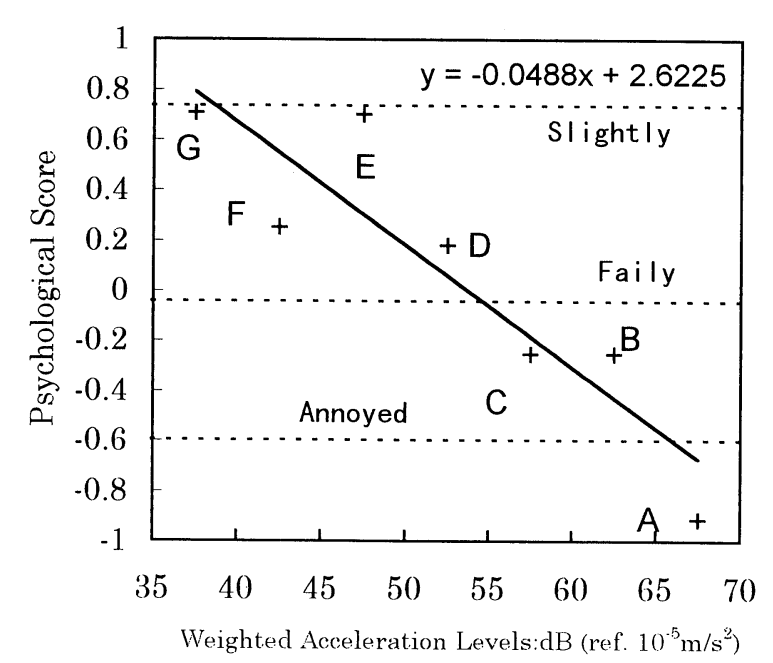

Site B

A:65-70dB, B:6(0-65dB, C:55-60)dB, D:50-55, E: 45-50dB.F: 4 ()-45dB, G:35-40

Fig. 10. Relationship between psychological score and weighted acceleration levels

a) In the stricken area, the vibration levels before and after the earthquake were almost the same.

b) Vibration levels at measured points within $50 \mathrm{~m}$ from Shinkansen tracks at Site A and Site B were almost the same, but at the 100-m point, the level at Site A was greater than that at Site B.

c) Indoor vibration levels were almost the same as outdoor vibration levels and vibration amplification due to house structure was also the same at both sites.

d) Most of the people living in the stricken area feel that the Shinkansen vibration increased after the earthquake.

e) According to the results of analysis by the "category judgment method", the vibration level at which $50 \%$ of the inhabitants complained about the Shinkansen vibration was approximately $54 \mathrm{~dB}$ at Site A and $50 \mathrm{~dB}$ at Site B.

f) If we take the results of Site B as a standard, people at Site A became approximately $4 \mathrm{~dB}$ more sensitive after the earthquake.

Therefore it can be concluded that the experience of the severe earthquake has caused people to be more sensitive to vibration.

Based on the results of this study, we would like to suggest that the psychological effects on people should be taken into consideration restoration plans and reconstruction work. It has pasted three years since the Hanshin earthquake hit this area. Many people have been disturbed by sideeffects.

\section{References}

1) Kobe University Department of Engineering Faculty of Civil Engineering South Hyogo Prefecture Seismology Investigation Group (1995 Feb.) The report of the South Hyogo Prefecture Earthquake Emergency Investigation (the first report).

2) Kobe University Department of Engineering Faculty of Civil Engineering South Hyogo Prefecture Seismology Investigation Group (1995 Mar.) The report of the South Hyogo Prefecture Earthquake Emergency Investigation (the second report).

3) Japan Industrial Standards (1990) Vibration level meter. JIS C 1510.

4) International Organization for Standardization (1990) Human response to vibration-measuring instrumentation. ISO 8041.

5) Japan Environmental Agency (1976) Recommendation-Measurement methods and regulation of Shinkansen vibration.

6) Guilford J.P. (1954) Psychometric methods. McGrawHill, New York.

7) Namba S, Kuwano S, Nakamura $T$ (1978) Rating of road traffic noise using the method of continuous judgement by category. SAJ 34, 29-34 (in Japanese).

8) Maeda S, Nakajima T (1984) A study by method of category judgment on evaluation of speech intelligibility. Journal of the Faculty of Science and Technology, Kinki Univ 20, 45-51 (in Japanese). 